\title{
EVALUATION OF A TRAINING INTERVENTION TO IMPROVE NOVICE DRIVERS' HAZARD MITIGATION BEHAVIOR ON CURVES
}

\author{
Jeffrey W. Muttart ${ }^{1}$, Ravi Agrawal ${ }^{2}$, Yalda Ebadi ${ }^{2}$, Siby Samuel ${ }^{2}$ \& Donald L. Fisher ${ }^{3}$ \\ ${ }^{1}$ Crash Safety Research Center LLC, ${ }^{2}$ University of Massachusetts Amherst, \\ ${ }^{3}$ Volpe National Transportation Center \\ Email:muttartj@gmail.com
}

\begin{abstract}
Summary: Newly licensed teenage drivers experience a higher risk of crashing compared to other age cohorts. Literature reveals that novice drivers exhibit poor hazard mitigation skills. The current study assesses the effectiveness of a training program at improving novice divers' hazard mitigation and speed selection behaviors on curves. In this study, drivers are randomly assigned to two training cohorts (ACT and placebo), and were exposed to 2 different scenarios of interest, one scenario contained a moderate curve left and the other included a tightening curve right. ACT trained drivers made more glances to the far extent of the curve, than the placebo-trained drivers. ACT (Anticipate, Control, and Terminate) trained drivers were also significantly more likely to slow to the target speed before the curve, when compared to the placebo trained drivers. The results indicate the effectiveness of ACT as a countermeasure, at training novice drivers to select better glancing and speed management strategies.
\end{abstract}

\section{INTRODUCTION}

Novice drivers aged 16 and 17 are approximately 50\% more likely to die in a crash than middleaged, experienced drivers aged 35-44 years old (FARS Query, 2012). Specifically, drivers younger than age 19 are much more likely to be involved in a single vehicle run-off-road crash than are more experienced drivers (Mayhew et al., 2003; Braitman et al., 2008). Drivers' speed selection before entering the curve is one of the major causes of single vehicle crashes at the curves. In most of the single vehicle crashes, the driver was found to have understeered, the likely result of braking sharply (Zegeer, et al, 1990) or over steering (Glennon., et al., 1983; Maeda., et al., 1977), producing a turn that was sharper than the highway curve and further reducing the available friction. Over steering and under steering occur after cresting the curve. Anticipating and slowing before the curve is the optimal solution to both problems (Glennon et al, 1983; Mikolajetz, 2009). Glennon et al. (1983) referred to the region three seconds before the curve, as the critical region of speed selection. In the region, drivers should begin to adjust both their speed and path. Such adjustments were particularly large on sharper curves.

To study why the novice driver has a higher crash rate than the experienced driver, it is important to differentiate between hazard anticipation and hazard mitigation. Pollatsek et al., (2006) found that the novice driver failed to both scan for the hazards, especially when the hazard is obscured, and then subsequently failed to appropriately mitigate the hazards. A simulator study showed that novice drivers failed to appropriately scan for the stop sign obscured by the bushes, and braked harder on average when approaching the stop sign compared to the experienced driver despite a "stop ahead" sign (Fisher et al., 2002). Also, when a driver failed to anticipate a hazard, they were more likely to fail to mitigate that hazard (Muttart., et al, 2013) Fisher et al. (2008) suggested that there are three components necessary for an effective novice driver-training curriculum: attention maintenance, hazard anticipation and lastly, speed and lane position management (referred to as risk mitigation). The current research evaluates the effectiveness of 
the third component of the curriculum, a training program referred to as ACT, which stands for anticipation, control and terminate. ACT builds upon RAPT (Risk and perception training) that has shown to be associated with reduced crash risk (Thomas, et al., 2016). ACT utilizes what has been learned with RAPT and extends the training to horn use, lane positioning, and slowing at specific periods at known crash scenarios. ACT training teaches speed selection behaviors in three broad categories of scenarios (intersections, curves and straight sections), of which only curves are of interest in the current paper. There were two curve types that were of prime interest in the current effort, and were examined. Left curves have been cited as being less of a threat with less speed loss (Mikolajetz, et al, 2009). Right curves, longer curves and curves with smaller radii have been associated with greater crash risk (Anderson, et al., 2000).

The current research examined driver glance and speed mitigation behaviors for gradual curves towards the left (less crash risk), and a longer tightening curve to the right (greater crash risk). It was hypothesized that, novice drivers who receive ACT training would be more likely to make earlier anticipatory glances toward the extent of the sightline. It was further hypothesized that, drivers who receive ACT training would begin to slow earlier than novice drivers. Finally, it was hypothesized that ACT trained drivers would slow more often if they avoided the crash than placebo trained drivers.

\section{METHOD}

\section{Participant}

Thirty-six newly licensed, novice drivers between 16 and 17 years old were recruited for this study. Half the participants were randomly assigned to the placebo training while the other half were assigned to ACT training. The mean age of those who received ACT training was 17.4 (SD $=0.3)$ years, while the placebo trained group had a mean age of $17.2(\mathrm{SD}=0.4)$ years. Drivers in both training groups had been licensed an average of 0.6 year. All participants in this study were recruited from the town of Amherst, MA and surrounding areas.

\section{Apparatus}

The simulator used for this experiment was a fixed-based driving simulator developed by Real time Technologies, consisting of a fully equipped Saturn sedan placed in front of three screens subtending 150 degrees horizontally and 30 degrees vertically. The images are displayed at a resolution of $1024 \times 768 \mathrm{dpi}$ in each screen with a refresh rate of $60 \mathrm{~Hz}$. The appropriate environment sounds and Doppler effects are produce by a surround sound system. To monitor and record eye movements and to determine the locations fixated by the drivers in the virtual world, a ASL Mobile Eye tracker was used. This head mounted, monocular eye tracker has an accuracy of approximately 0.5 degrees of visual angle.

\section{Training programs}

In this study, two training programs were administered: the ACT and a placebo.

ACT training program. ACT program is a rule-based program consisting of a practice module, a pretest module, a training and mediation module, and a posttest module. In each of the modules, a series of snapshots leading up to a traffic scene were shown to participants. In all modules, to indicate the location of the glance, the participants were asked to identify potential threat area by clicking on them, using the mouse pointer. In the practice module, participants were first shown the various scenarios with the snapshots appearing at the rate of one frame per second besides which, they got introduced to the use of foot pedal position selectors, horn selector, and the lane 
position selectors and allowed to practice (depicted in Figure 1). As with RAPT, the user also had the option of making anticipatory glances (mouse clicks on the forward scene) (Fisher et al., 2008). In the pretest module, the drivers were shown nine driving scenarios including the eight most common fatal crash configurations according to the Fatality Analysis Reporting System, which included three curves (Figure 1). The other scenarios were 3 intersections and 3 straight section road segments. In the current paper, only the effectiveness of the ACT training on novice drivers' speed selection behavior at curves is examined. In the training module, the participants learned through error based training, they had opportunity to make mistakes, and they also got introduced to how to correct those mistakes. For mediation, a rule-based method was taught to the participants and the participants also learned how to understand the training of rules and exceptions to rules. In the post-test module, all drivers were assessed on the same 9 scenarios that were shown in the pre-test and training modules.

Driving "rules" were based upon the performances of exemplary drivers at the same locations that is reported in previous research (Muttart et al., 2013). The exemplary drivers were ages 2661 with no crashes or violations in the previous ten years. As an example, should a novice driver select the brake (red) button when 1 second before the apex of the curve, they would be told during the mediation training that the exemplary driver group usually applied the brake at 3 seconds before the apex of the curve.

\begin{tabular}{|l|l|}
\hline Driver's View of Training Scenario & Description \\
\hline & \begin{tabular}{l} 
1. $\begin{array}{l}\text { Curve right with roadside pedestrians } \\
\text { left } \\
\text { Most like: Curve 1 (Curve left) } \\
\text { Drivers negotiate a curve right with } \\
\text { possibility of oncoming vehicle crossing } \\
\text { centerline. }\end{array}$ \\
\hline
\end{tabular} \\
\hline
\end{tabular}

Figure 1: The 3 curve training scenarios from ACT program (The pedals at the bottom right represent the throttle - green when selected, "off-throttle" - small pedal between brake and throttle which appears yellow when selected, and brake - which is red when selected as pictured. The five arrows represent positions left and right of lane-center. The horn icon shows where the horn is located on a steering wheel that is overlaid on the lower portion of the screen (not pictured) and users may click anywhere in the forward view to indicate where they wish to glance.) 
The ACT training module consisted of three submodules delivered always in the order presented here. First submodule is the Safety Bubble, in which drivers were taught to position the vehicle within the lane, and they were also taught that they should sound the horn when there was a situation where they believed other drivers might not know or see their location (Figure 2). Lastly, as a means of staying away from other vehicles, they should slow (if a slower speed was not already selected) for HRECCS (Hidden hazards and roadside obstacles). HRECCS

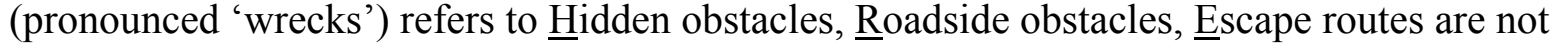

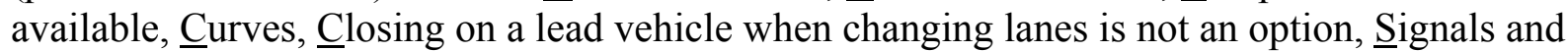
warnings. The second submodule is HRECCS, a driver learns that, it is important to brake, slow or change lane position or sound the horn in case a HRECCS is present. The third submodule is a feedback submodule in which the nine scenarios were trained one at a time. In the feedback submodule, the novice drivers were shown aerial views depicting the potential hazards that existed at each scenario following which there was a glance mitigation explanation component where participants were taught why a hidden hazard was a threat. Drivers were taught to glance to the extent of the sight line, move toward the outside of the curve (left when approaching a right curve), slow, and sound the horn and slow for a curve, but a driver should brake if any two HRECCS exist. The drivers are shown a curve (C) and a road side pedestrian (R). An individual feedback component involving feedback for incorrect responses completed the feedback submodule.

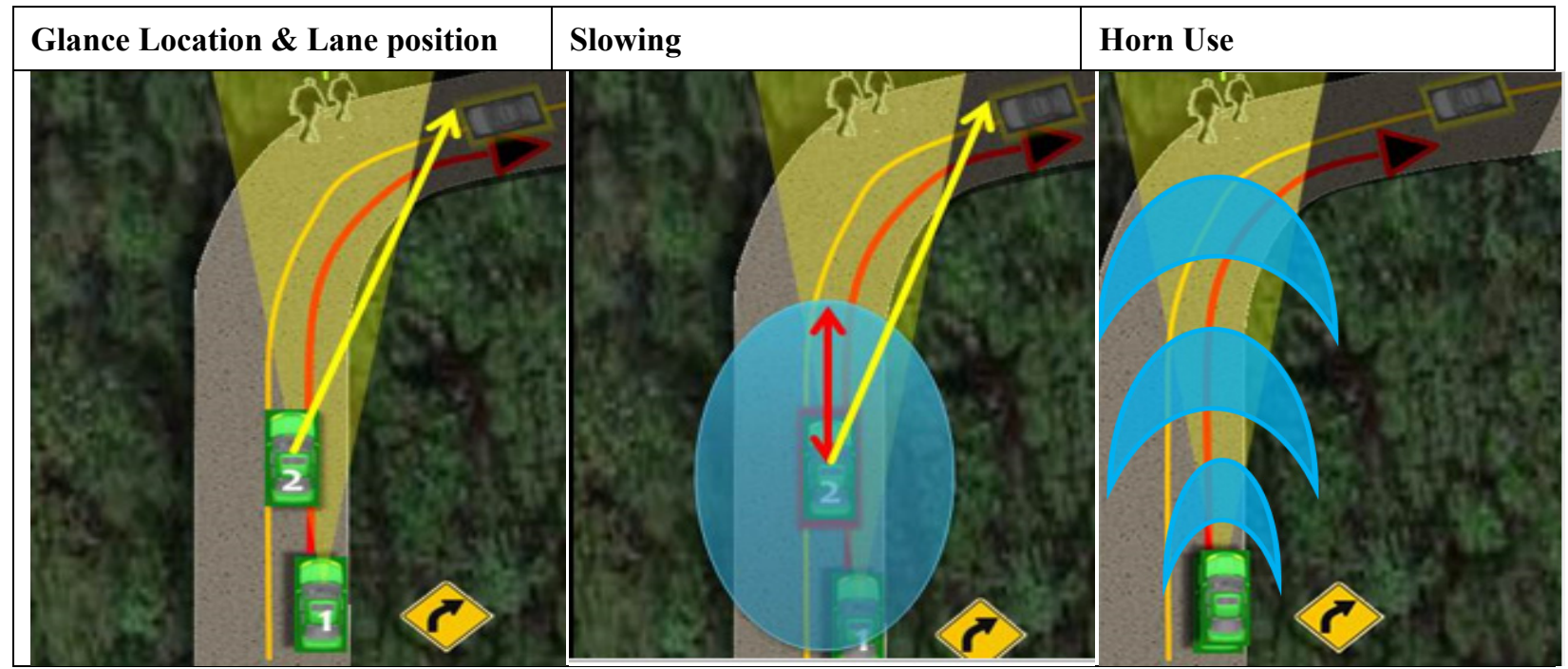

Figure 2: The three training slides for ACT Scenario 1, Here, the drivers were taught about the concepts of hazard anticipation (glancing toward latent hazards), Slowing for HRECCS (Hidden hazards and roadside obstacles), maintaining a Safety Bubble by changing lane position (third slide), and using the horn when possible (when not steering) to keep others out of the Safety Bubble.

\section{Placebo Training Program}

The ACT and placebo versions of the program appear similar except training components are different. In the placebo program, there are questions regarding signs and drivers should answer how they react to objects which appear at traffic scenes, like a reaction time tester.

\section{Experimental Design \& Driving Scenarios}

Drivers were randomly assigned to one of two training programs: half of the drivers were assigned to the placebo training, while the other half were assigned to ACT training. At one curve, drivers negotiated a gradual curve to the left and in the other curve, drivers had to 
negotiate a tightening curve to the right and approach a car that is parked partially on the road. The ordering of scenarios was counterbalanced across subjects.

\section{Procedure}

Upon arrival to the lab, participants were asked to read and sign an informed consent. A preexperimental questionnaire, including the training program was given to drivers from both the ACT-trained and placebo-trained groups. Drivers were then outfitted with an eye tracker, had their eyes calibrated and were provided a practice drive in the simulator to get them acclimated with the controls of the simulator cab and the simulated environment. Following this, the participants navigated the curve scenarios while driving for three 18 minutes drives in the simulator.

\section{Dependent Variables}

Anticipatory glances, slowing and lane positions were recorded for at least ten seconds before a curve and the scoring was binary. Anticipatory glances were defined as glances toward the far extent of the sight line around the curve and were based upon blind scorers who received 90 minutes of training. The ACT trained and the placebo trained drivers were evaluated on whether anticipatory glances were made when approaching curves. Specifically, anticipatory behaviors of glancing to the far extent, slowing to target speed and moving within the lane were compiled. Every one second, the averages of three samples (total 0.1 second in length) were taken from the simulator information for throttle position (on or off), brakes on or off, and vehicle speed. Target speed is a measure of anticipation in that it equaled the current speed minus the product of the time to impact at that speed and the change in speed during the previous second. Thus, target speed equals the expected speed at the apex of the curve from any point before the curve. The target speed thresholds were selected using the curve speed models proposed by Bonneson et al. (2009).

$$
S_{\text {target }}=S_{0}-t t i \times \Delta S_{0-1}
$$

\section{RESULTS}

The results presented include aggregate glance, braking and slowing behaviors along with conditional slowing and crashes.

Aggregate Glance, Braking and Slowing Behaviors

When approaching a sharp curve to the right (see Figure 3), the ACT trained drivers were significantly more likely to make a glance to the far extent when five and six seconds before the curve $[\mathrm{t}(35)>2.28 ; \mathrm{p}<0.03]$. At the sharp curve, right, ACT trained drivers slowed to target speed of $20 \mathrm{mph} 2.7 \mathrm{~s}$. ( $\mathrm{SD}=0.4 \mathrm{~s}$.) before the curve. Placebo trained drivers did not reach target speed until $1.8 \mathrm{~s}$. ( $\mathrm{SD}=1.7 \mathrm{~s}$.) before the curve, a difference which was statistically significant $[\mathrm{t}$ $(29)=2.36, P=0.03]$. At the gradual curve left, both the group ACT trained drivers and placebo trained driver glanced towards the far extent $3 \mathrm{~s}$ before the curve however, the differences were not statistically significant $(\mathrm{t}(35)=0.14 ; \mathrm{p}=0.89)$. Related to the mitigation behavior in the curve left, both ACT and placebo trained groups were equally likely to slow to the target speed of the 34-mph, $3 \mathrm{~s}$ before the curve $(\mathrm{t}(35)=1.17 ; \mathrm{p}=0.26)$.

\section{Conditional Slowing and Crashes}

The conditional outcomes across the two groups were compared in two ways. First, the percentage of drivers who correctly scanned towards the far extent and slowed to target speed, given that they crashed or not was examined. Second, the percentage of drivers who slowed to 
target speed, given that they glanced to the far extent was compared for the two groups. At the sharp curve right, the ACT trained driver were five times more likely to make a glance to the far extent (11 vs. 2) and 1.16 times more likely to slow to the target speed of 20-mph (14 vs 12). Importantly, 9 ACT trained drivers both glanced and slowed whereas only 2 Placebo trained drivers both glanced and slowed. At the gradual curve left, the ACT trained drivers were less likely to make a glance (12 vs 14 ) and only 1.19 times more likely to slow than the placebo drivers (31 vs 26). Overall 11 ACT trained drivers both glanced and slowed whereas only 8 Placebo trained drivers both glanced and slowed. One ACT trained driver and five placebo trained drivers crashed at the sharp right curve location but there were no crashes at the moderate curve. Of the ACT and placebo drivers that crashed, no driver made a glance to the far extent when five to eight seconds before the curve. None of the placebo trained drivers that crashed had slowed to the target speed.

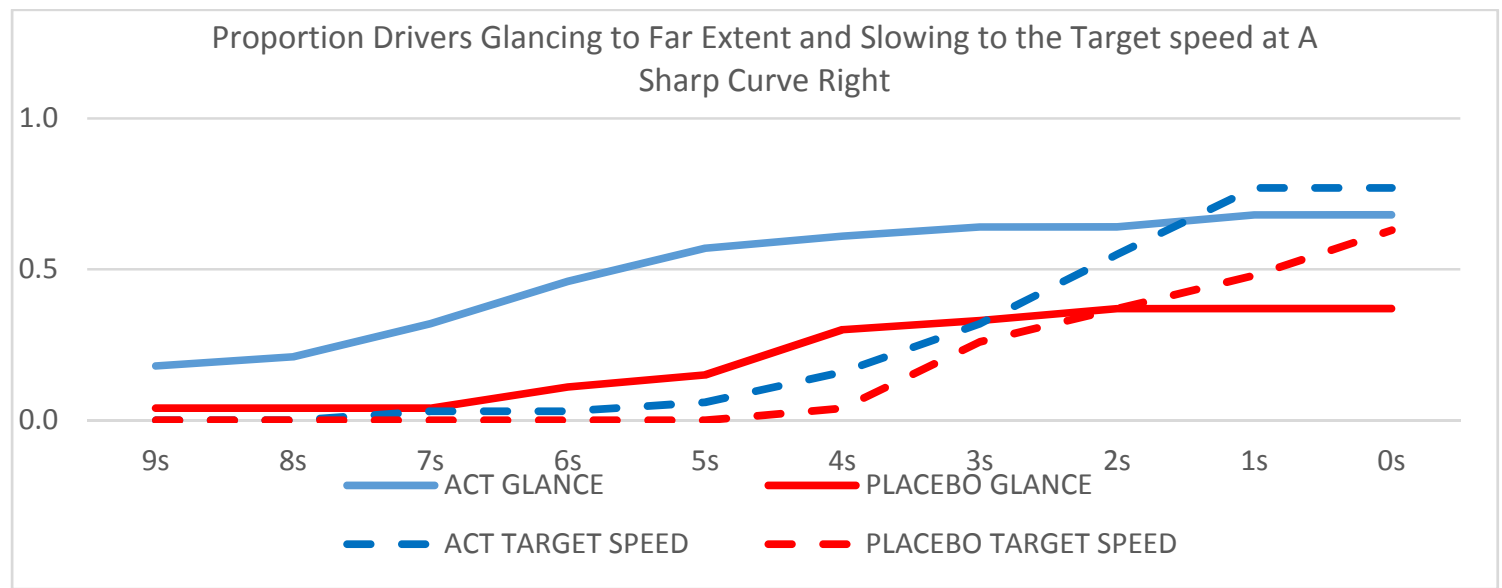

Figure 3. Glancing and speed selection of the ACT trained drivers and Placebo trained drivers when approaching a sharp curve to the right [Blue lines: Proportion of the driver who glanced to the far extent, Red Lines: Proportion of driver who slowed to the target speed of $32 \mathrm{~km} / \mathrm{h}(20 \mathrm{mph})]$

On the sharp right curve, the percentage of drivers that crashed among those who slowed versus those who did not slow was compared. ACT trained drivers were much less likely to crash if they had slowed to the target speed, $\mathrm{P}(\mathrm{C} \mid \mathrm{S})=0.07$ versus $\mathrm{P}(\mathrm{C} \mid \mathrm{nS})=0.25[\mathrm{t}(35)=1.1 ; \mathrm{p}=0.29]$. But placebo trained drivers were significantly less likely to crash if they slowed to target speed before the curve, $\mathrm{P}(\mathrm{C} \mid \mathrm{S})=0.0$ versus $\mathrm{P}(\mathrm{C} \mid \mathrm{nS})=0.83[\mathrm{t}(35)=9.49 ; \mathrm{p}=0.00]$.

\section{SUMMARY \& DISCUSSION}

Comparing the ACT trained driver and placebo trained drivers based on glancing and slowing as a conditioning event, ACT trained drivers made more glances to the far extent than the Placebo trained driver. ACT trained drivers also reduced their speed by the amount predicted by the Mikolajetz or Bonneson speed loss models (Bonneson \& Pratt, 2009, Mikolajetz et al., 2009). At the sharp curve, ACT trained drivers were significantly more likely to glance towards the far extent when five to seven seconds from the curve. The difference between the ACT trained and Placebo trained drivers who slowed to the 20-mph target speed was between 14 and 29 percentage points. At the moderate curve, the difference between the ACT trained driver and Placebo trained driver who glance to the far extent was 14 percentage points. From a mitigation perspective, ACT trained drivers were significantly more likely to slow to the target speed of 20 mph when six to eight second before the curve, compared to the placebo trained drivers. 
When examining conditional probabilities, slowing was not always associated with glances. At the right curve, $89 \%$ of the ACT trained drivers and $100 \%$ of the placebo trained drivers who appropriately glanced, slowed to the target speed of $20 \mathrm{mph}$. Clearly, slowing was associated with an anticipatory glance. However, only two of the 18 placebo-trained drivers glanced to the far extent. When there was not a glance to the far extent, the percentage of drivers that slowed dropped to $71 \%$ for the ACT drivers and $62 \%$ for the placebo trained drivers. The results for the ACT trained drivers are similar to those observed from the experienced, exemplary drivers in Muttart et al, 2013. At the curve left, while similar numbers of ACT trained and placebo trained drivers made glances to the far extent, many more ACT trained drivers utilized the information gained, and slowed. Of those who glanced to the far extent at the curve left, $92 \%$ of the ACTtrained drivers slowed to the target speed of $34 \mathrm{mph}$ compared to only $57 \%$ of the placebo trained drivers. These results are better than those from the experienced drivers in Muttart et al, 2013.

Recommendations for further research include validation with open road driving to examine transfer to real-world driving. Also, this research did not examine the long-term crash rates of the ACT-trained versus placebo-trained drivers. However, the initial results appear promising at improving novice drivers' speed selection behavior. Training programs like ACT may be used as an effective countermeasure to improve novice driver behavior.

\section{ACKNOWLEDMENTS}

Portions of this research were funded by grants from the National Institutes of Health (1R01HD057153-01), the National Science Foundation (Equipment Grant SB 9413733 for the partial acquisition of the driving simulator) and the Arbella Insurance Charitable Foundation.

\section{REFERENCES}

Anderson, I. B., \& Krammes, R. A. (2000). Speed reduction as a surrogate for accident experience at horizontal curves on rural two-lane roads, Transportation Research Record 1701, Washington, DC: Transportation Research Board.

Bonneson, J. A., \& Pratt, M. P. (2009). A model for predicting speed along horizontal curves on two-lane highways, Paper No. 09-1419, Washington, DC: Transportation Research Board.

Braitman, K. A., Kirley, B. B., McCartt, A. T., \& Chaudhary, N. K. (2008). Crashes of Novice Teenage Drivers: Characteristics and Contributing Factors. Journal of Safety Research, 39 (1), 47-54.

Fatality Analysis Reporting System [FARS]. (2012). (National Highway Traffic Safety Administration) Retrieved November 12, 2012, from http://www.nhtsa.gov/people/ncsa/fars.html

Fisher, D. L., Laurie, N. E., Glaser, R., Connerney, K., Pollatsek, A., Duffy, S. A. \& Brock, J. (2002). The use of an advanced driving simulator to evaluate the effects of training and experience on drivers' behavior in risky traffic scenarios. Human Factors, 44, pp. 287-302.

Fisher, D. L. (2008). Evaluation of PC-Based Novice Driver Risk Awareness DOT HS 810926. Washington, DC: National Highway Traffic Safety Administration.

Glennon, J. C., Neuman, T. R., Leisch, J. T. (1986). Safety and operational considerations for design of rural highway curves, (DOT - FH - 11-9575). McLean, VA: Federal Highway Administration. 
Maeda, T., Irie, N., Hidaka, K., Nishimura, H. (1977). Performance of driver-vehicle system in emergency avoidance, (Technical paper 770130). Warrendale, PA; Society of Automotive Engineers.

Mayhew, D. R.; Simpson, H. M. \& Pak, A. (2003). Changes in collision rates among novice drivers during the first months of driving. Accident Analysis and Prevention, 3, 683-91.

Mikolajetz, A., Henning, M. J., Tenzer, A., Zobel, R., Krems, J. F., Petzoldt, T. (2009). Curve negotiation: identifying driver behavior around curves with the driver performance database, Proceedings of the fifth International Driving Symposium on Human Factors in Driver Assessment, Training and Vehicle Design, 391 - 397.

Muttart, J. W., Fisher, D. L., Pollatsek, A. P., \& Marquard, J. (2013). Comparison of anticipatory glancing and risk mitigation of novice drivers and exemplary drivers when approaching curves. In Proceedings of the Seventh International Driving Symposium on Human Factors in Driver Assessment, Training, and Vehicle Design (pp. 212-218). Public Policy Center, University of Iowa Lake George, NY. Iowa City.

Pollatsek, A., Fisher, D. L. \& Pradhan, A. K. (2006). Identifying and Remediating Failures of Selective Attention in Younger Drivers. Current Directions in Psychological Science, 15, 255-259.

Thomas, F. D., S. L. Rilea, R. D. Blomberg, R. C. Peck. \& K. T. Korbelak, Evaluation of the Safety Benefits of the Risk Awareness and Perception Training Program for Novice Teen Drivers. DOT HS 812 235. 2016. Washington, D.C: National Highway Traffic Safety Administration.

Zegeer, C., Stewart, R., Reinfurt, D., Council, F.M., Neuman, T., Hamilton, E., Miller, T., \& Hunter, W. (1990). Cost Effective Geometric Improvements for Safety Upgrading of Horizontal Curves. Publication No. FHWA-RD-90-021, University of North Carolina, Highway Safety Research Center, Chapel Hill, NC. 